\title{
MiR-138-5p targets RUNX2 to inhibit osteogenic differentiation of aortic valve interstitial cells via Wnt/ $\beta$-catenin signaling pathway
}

Fei Yan ${ }^{1 *}$, Qiang Huo ${ }^{1}$, Weimin Zhang ${ }^{1}$, Tingting $\mathrm{Wu}^{2}$, Daniyaer Dilimulati ${ }^{1}$ and Lin Shi $^{1}$

\begin{abstract}
Background: Human aortic valve interstitial cells (hAVICs) are a key factor in the pathogenesis of calcific aortic valve disease (CAVD). This research examines the role and mechanism of microRNA miR-138-5p in osteogenic differentiation of hAVICs.

Methods: RT-qPCR analysis was applied for detecting miR-138-5p and RUNX2 expression in valve tissues of CAVD patients and controls. On completion of induction of osteogenic differentiation of hAVICs, and after overexpression or interference of miR-138-5p expression, the condition of osteogenic differentiation and calcification of hAVICs was confirmed using alkaline phosphatase staining and alizarin red staining. Subsequently, western blot was utilized to detect the expression of osteogenesis-related proteins OPN and ALP, and Wnt/ $\beta$-catenin signaling pathway-related proteins. Finally, the relationship between miR-138-5p and RUNX2 was validated by dual-luciferase reporter assay and Pearson's correlation test.
\end{abstract}

Results: Down-regulation of miR-138-5p was found in CAVD patients and during osteogenic differentiation of hAVICs. Overexpression of miR-138-5p contribute to the inhibition of osteoblast differentiation and calcium deposition in $\mathrm{AAVICS}$, and of ALP and OPN protein expression. RUNX2 was a target gene of miR-138-5p, and it was negatively correlated with miR-138-5p in CAVD. Additionally, overexpression of RUNX2 could reverse the inhibitory effect of miR138-5p on osteogenic differentiation of hAVICS.

Conclusion: miR-138-5p can act as a positive regulator of osteogenic differentiation in CAVD patients to involve in inhibiting valve calcification, which is achieved through RUNX2 and Wnt/ $\beta$-catenin signaling pathway.

Keywords: Calcific aortic valve disease (CAVD), Aortic valve interstitial cells (AVICs), Osteogenic differentiation, miR138-5p, RUNX2, Wnt/ß-catenin

*Correspondence: yfheart@163.com

1 Department of Cardiac Surgery, The First Affiliated Hospital

of Xinjiang Medical University, 137 Liyushan South Road, Xinshi District,

Urumqi 830054, Xinjiang, China

Full list of author information is available at the end of the article

\section{Introduction}

Calcific aortic valve disease (CAVD), with the prevalence ranking first among valvular heart diseases clinically, is characterized by valve thickening and calcification, aortic hemodynamic disorders and heart failure, which is mainly responsible for valve replacement failure. Among the numerous etiological factors of CAVD, the osteogenic differentiation of aortic valve 
interstitial cells (AVICs) that mainly regulate aortic valve structure and function, and subsequent calcium deposition are considered to be key factors [1-4].

MicroRNAs (miRNAs) refer to small non-coding RNA molecules which are highly conserved and single-stranded. They mediate the levels of their target genes through transcriptional repression or mRNA degradation, thus regulating a variety of physiological and pathological processes [5], including cell proliferation, apoptosis [6], inflammation [7], and cancer [8]. In recent years, many miRNAs involved in osteogenic differentiation of AVICs have been identified. For example, miR-29b activates wnt $3 / \beta$-catenin/Smad3 signaling to inhibit TGF- $\beta 3$ and consequently to promote AVICs calcification [9], while miR-638 targets Sp7 to inhibit human AVICs (hAVICs) calcification by targeting Sp7 [10]. Among miRNAs, regulation of miR-138-5p in various biological processes has been proved, such as its involvement in developmental events associated with cell differentiation. For example, osteogenic differentiation of human mesenchymal stem cells (hMSCs) can be negatively regulated by miR-138-5p; this miRNA mediates AK-ERK1/2 activity, thereby decreasing Runx2 expression and achieving the negative regulation [11]. By contrast, this process can be promoted by miR138-5p knockdown and the resulting up-regulation of FOXC1 [12]. However, what and how miR-138-5p affects CAVD has not been reported. Therefore, this study first clarifies miR-138-5p expression during osteogenic differentiation of hAVICs, and then its specific function and mechanism of miR-138-5p in this process are confirmed by cell experiments. The findings of this research provide a potential strategy for the prevention or treatment of multiple valvular calcification-associated diseases.

\section{Materials and methods}

\section{Collection of human aortic valve tissue}

Human aortic valve tissue $(n=10)$ were obtained from calcified aortic valves of adult patients undergoing aortic valve replacement in our hospital, and non-calcified aortic valves of patients with aortic annular dilatationcaused aortic regurgitation $(n=5)$. Pathomorphologic diagnosis were confirmed at the time of aortic valve replacement. Patients with a history of infective endocarditis or rheumatic heart disease were excluded. All patients had signed informed consent. The study was approved by the ethics review board of our hospital and conformed to the principles specified in the Declaration of Helsinki, with an approval by the Ethics Committee of The First Affiliated Hospital of Xinjiang Medical University (K202107-09).

\section{Isolation and culture of hAVICs}

hAVICs were isolated as described by Zhang et al. [2]. First, on completion of aseptic acquisition of the aortic valve from calcified aortic valves and normal controls, endothelium and non-lobular tissue were removed from the ventricles and aorta. Subsequently, the lobule was soaked in $0.25 \%$ trypsin for $5 \mathrm{~min}$ and then cut into $3 \times 3 \mathrm{~mm}$ pieces. Next, after $12 \mathrm{~h}$ digestion of the pieces by trypsin at ambient temperature, the digested cells were collected and transferred to DMEM medium (Sigma, USA). The medium containing L-glutamine, 10\% fetal bovine serum (FBS), $10 \mathrm{U} / \mathrm{L}$ penicillin, and $10 \mu \mathrm{g} / \mathrm{L}$ streptomycin was placed in an incubator $\left(5 \% \mathrm{CO}_{2}, 37^{\circ} \mathrm{C}\right)$, and changed every 3 days. The cells were examined microscopically for purity after five passages.

\section{Transfection of hAVICs}

hAVICs were plated in 6-well plates with a density of $5 \times 10^{4}$ cells/well until reaching $60-70 \%$ confluence. After that, $2 \mathrm{nM}$ miR-138-5p mimics, miR-138-5b inhibitor or control were transfected into cells using Lipofectamine2000 transfection kit (Invitrogen), respectively. Then with low serum medium (contained 1\% FBS, changed every 3 days) for culturing the transfected cells, cells were collected after 21 days for subsequent analysis.

\section{Osteogenic differentiation induction of hAVICs}

hAVICs were plated in 24-well plates with $4 \times 10^{4}$ cells in each well until reaching $60-70 \%$ confluence. After that, osteoblastic differentiation was induced by continuous culture in medium containing $50 \mathrm{mg} / \mathrm{mL}$ ascorbate2-phosphate and $10 \mathrm{mM} \beta$-glycerophosphate for 21 days, with the culture in blank medium as a control. On the 7th day and 21st day of induction, alkaline phosphatase (ALP) staining and alizarin red staining were performed to evaluate osteoblast induction.

\section{Alizarin red staining}

Primary hAVICs cultured in medium containing $10 \mathrm{mM}$ $\beta$-glycerophosphate were fixed in $70 \%$ ethanol for $1 \mathrm{~h}$ at ambient temperature and then stained with $40 \mathrm{mM}$ alizarin red (Beyotime, China) for $10 \mathrm{~min}$. On completion staining, cells were rinsed with PBS to clear nonspecific staining, and the stained matrix was photographed using a digital microscope. Afterwards, the alizarin red S dye was released from the cell matrix by incubation in cetyl pyridinium chloride for $15 \mathrm{~min}$, followed by staining quantification of the amount of the released dye by spectrophotometry at $540 \mathrm{~nm}$. Finally, the quantitative results were normalized to the total cellular protein content [13]. 


\section{Alkaline phosphatase (ALP) staining}

After osteogenic induction of hAVICs and removal of the original medium, the cells were rinsed by PBS, and then stained with an appropriate amount of BCOP/NBT staining solution (Thermo, USA) for $25 \mathrm{~min}$ at room temperature in the dark. On completion staining, cells were rinsed with $\mathrm{PBS}$ to clear nonspecific staining, and the stained matrix was photographed using a digital microscope. Afterwards, $400 \mu \mathrm{L}$ of cell lysate was added to each well, and finally the cell supernatant was collected after centrifugation $(1000 \mathrm{~g}, 10 \mathrm{~min})$.

\section{Detection of alkaline phosphatase (ALP) activity}

Detection of ALP activity of hAVICs was completed by utilizing an alkaline phosphatase activity colorimetric assay kit (BioVisionInc., Mountain View, CA). On completion of rinsing step by PBS, transfected cells were added with $50 \mathrm{ml}$ of buffer to homogenize. After that, the insoluble materials were removed through centrifugation $(13,000 \mathrm{~g}, 3 \mathrm{~min})$, and the samples were plated to a 96-well plate in equal volumes at different concentrations. Then, with $5 \mathrm{mM}$ of $50 \mathrm{~mol} / \mathrm{l} \mathrm{pNPP}$ solution in each control well and test well, an incubation for a period of $60 \mathrm{~min}$ was performed at ambient temperature in the dark. Finally, based on the standard curve, ALP activity was calculated: ALP activity $(\mathrm{U} / \mathrm{mL})=\mathrm{A}$ (amount of pNP produced by each sample, $\mu \mathrm{mol}$ ) $/ \mathrm{V}$ (volume of the reaction system, $\mathrm{mL}$ )/ $\mathrm{T}$ (time of reaction, $\mathrm{min}$ ).

\section{RT-qPCR}

TRizol reagent (Invitrogen, USA) was adopted to extract the total RNA from hAVICs, and Toyoba Reverse transcription kit (Fermentas, Canada) for reverse transcription from RNA to cDNA. Subsequently, ABI PRISM7900 Sequence Detection System (Applied Biosystems, Foster City, CA) was employed in RT-qPCR. Ultimately, with GAPDH and U6 as internal according to the instructions. GAPDH gene and U6 were used as reference genes, relative quantification of target gene expression was completed by $2^{-\Delta \Delta \mathrm{Ct}}$ method (Table 1 ). Three independent experiments were in 3 replicates.

\section{Dual-luciferase reporter assay}

The binding site of miR-138-5p and RUNX2 gene was predicted utilizing TargetScan database (http://www. targetscan.org/vert_72/). On the completion of the construction of RUNX2-WT and RUNX2-MUT vectors, 293T cells cultured in 24-well plates were transfected with these vector and miR-138-5p mimics by using Lipofectamine2000 (Invitrogen), respectively. The cells were collected $48 \mathrm{~h}$ after transfection, and lysed using lysate (Beyotime, China), followed by measurement of
Table 1 Primer sequences

\begin{tabular}{ll}
\hline Gene & Sequence $\left(\mathbf{5}^{\prime} \mathbf{-} \mathbf{3}^{\prime}\right)$ \\
\hline miR-138-5p & F AGCTGGTGTTGTGAATCAGGCCG \\
U6 AACGCTTCACGAATTGCGT & F CTC GCTTCGGCAGCACA \\
R AACGCTTCACGA ATTTGCGT \\
FUNX2 \\
FATGGCATCAAACAGCCTCTTCAGC \\
RAPDH \\
\end{tabular}

luciferase activity with the dual luciferase reporter assay system (Promega). Each group is provided with two parallel wells, and the experiment was in 3 replicates.

\section{Western blot}

Total protein was extracted from hAVICs using the Radio immunoprecipitation assay (RIPA), and the protein concentration was determined by a BCA kit (Thermo, USA). Then after separation using 10\% SDS-PAGE, the proteins were blotted onto PVDF membranes at $200 \mathrm{~mA}$, followed by $1 \mathrm{~h}$ blocking with $5 \%$ skimmed milk at room temperature. Subsequently, the membranes were incubated with antibodies against RUNX2, Wnt5a, $\beta$-catenin, OPN, ALP and $\beta$-actin antibodies overnight at $4{ }^{\circ} \mathrm{C}$. After washing with PBST for 3 times, the membranes were then incubated with secondary antibodies at room temperature for $2 \mathrm{~h}$ and rinsed with PBST for three times again. Finally, an exposure apparatus was used to develop proteins, and ImageJ software for grayscale analysis. Relative protein expression was calculated using $\beta$-actin as a control.

\section{Statistical analysis}

SPSS 21.0 software was utilized for statistical analysis, and data were expressed as mean \pm standard deviation (SD). Student's $t$-test was performed for two groups comparisons. One-way analysis of variance (ANOVA) followed by multiple comparisons was carried out. A statistically significant difference can be suggested if $P<0.05$.

\section{Results}

miR-138-5p affects osteogenic differentiation of hAVICs

First, by using RT-qPCR for determining miR-138-5p expression in the valve tissues of CAVD patients, significantly lower miR-138-5p was identified in the CAVD patients compared with the controls (Fig. 1A). Further, cell experiments confirmed what effects miR-138-5p had on osteogenic differentiation and calcification of hAVICs. As shown in Fig. 1B, miR-138-5p was lowly expressed 


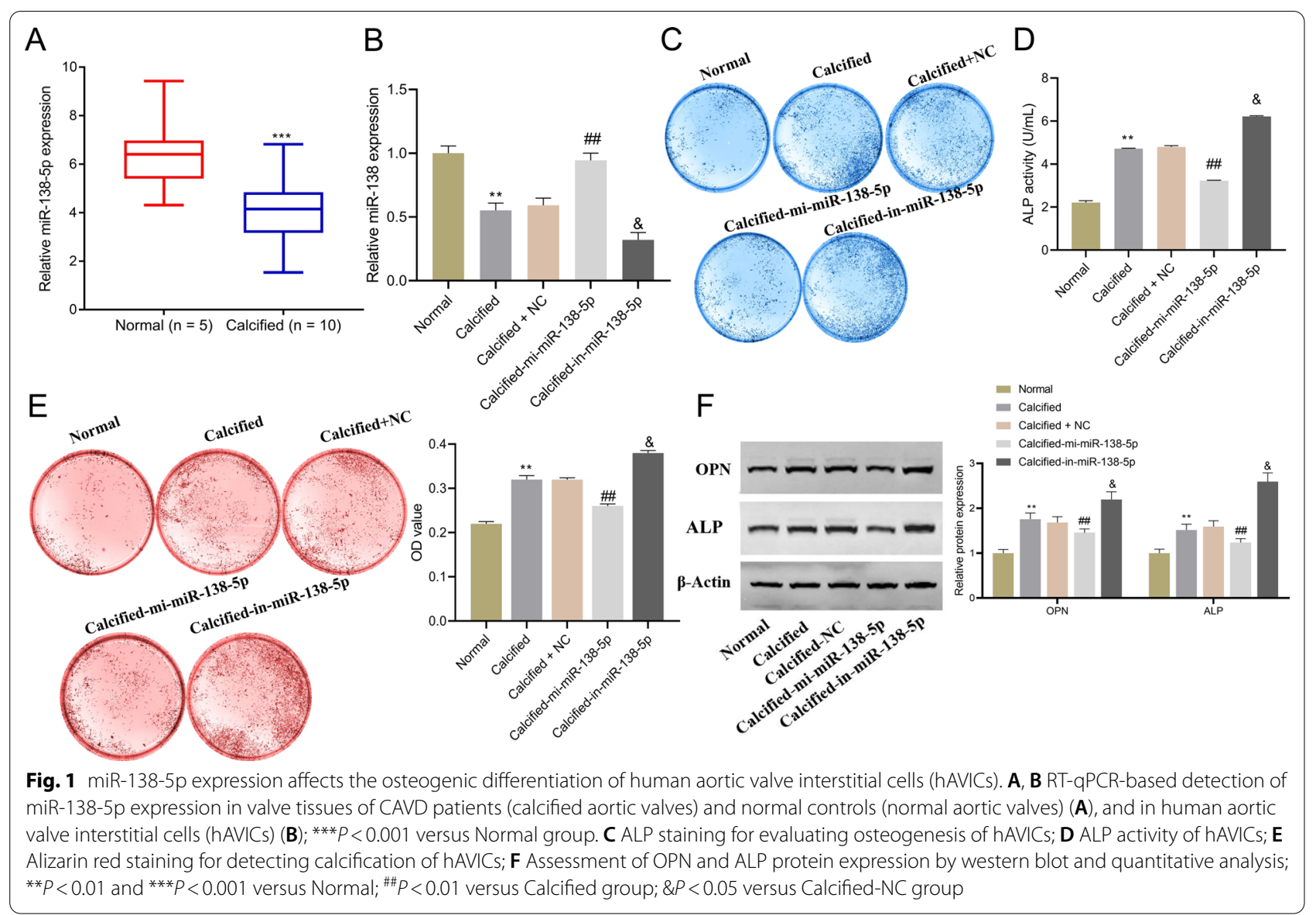

during osteogenic differentiation of hAVICs. Up-regulation of miR-138-5p expression by transfection of miR138-5p mimics and down-regulation of its expression by miR-138-5p inhibitor, suggested successful transfection (Fig. 1B). In addition, overexpression of miR-138-5p was responsible for the inhibition of ALP activity and calcification in hAVICs (Fig. 1C-E), and expression of osteogenic differentiation markers (osteopontin (OPN) and ALP proteins) (Fig. 1F). By contrast, inhibiting miR138-5p could achieve opposite results. Collectively, miR138-5p was downregulated in osteogenic differentiation of hAVICs, while overexpressed miR-138-5p could significantly inhibit osteogenic differentiation and calcification of hAVICs.

\section{MiR-138-5p targets RUNX2 in CAVD}

How miR-138-5p inhibited osteogenic differentiation of hAVICs was further investigated. TargetScan database predicted the binding site between RUNX2 and miR-138-5p, and the prediction was confirmed by dualluciferase reporter assay (Fig. 2A). In comparison with the controls, significant up-regulation of both RUNX2 mRNA and protein was found in the valve tissues of
CAVD patients (Fig. 2B, C). During osteogenic differentiation of hAVICs, overexpressed miR-138-5p inhibited RUNX2 mRNA and protein expression, while miR-138-5p knockdown achieved an opposite result (Fig. 2D, E). Further correlation analysis indicated a negative correlation between RUNX2 and miR-138-5p expression in CAVD (Fig. 2F).

\section{MiR-138-5p affects osteogenic differentiation of hAVICs by targeting RUNX2}

Whether RUNX2 involved in the mechanism of miR138-5p affecting osteogenic differentiation of hAVICs was investigated by cell rescue assays. Specifically, in comparison with Calcified-NC group, RUNX2 expression in the Calcified-mi-miR-138-5p group was significantly lower, while an increase of RUNX2 expression was found in the Calcified + mi-miR-138-5p + RUNX2 group (Fig. 3A). Overexpressed RUNX2 relieved the inhibitory effect of up-regulation of miR-138-5p on ALP activity and calcification in hAVICs (Fig. 3B-D), while it increased OPN and ALP protein expression (Fig. 3E). Taken together, the inhibitory effect of overexpressed 


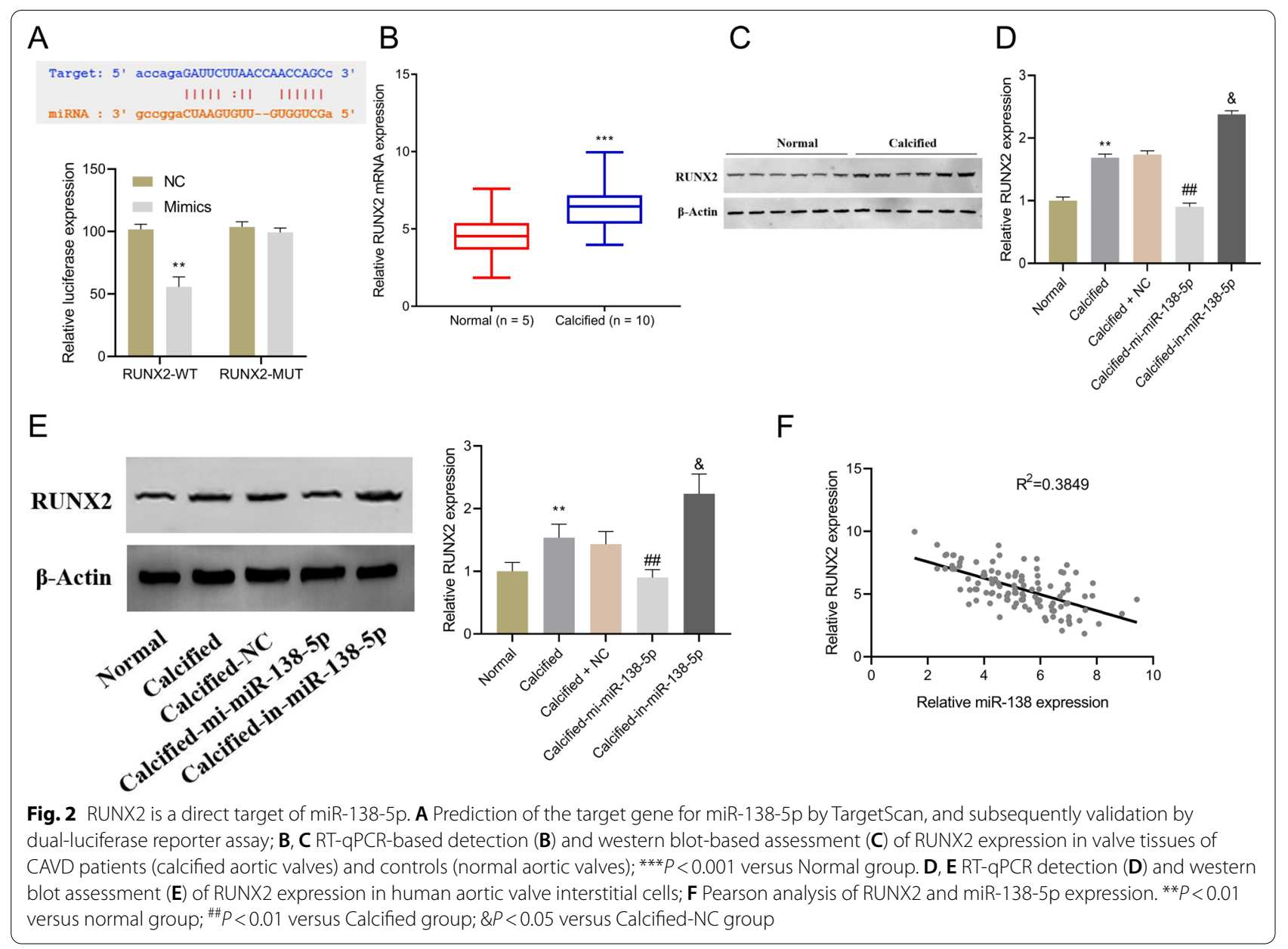

miR-138-5p on osteogenic differentiation was achieved by decreasing RUNX2.

\section{MiR-138-5p regulates RUNX2-Wnt/ $\beta$-catenin axis to inhibit osteogenic differentiation of $h A V I C s$}

Involvement of Wnt/ $\beta$-catenin signaling pathway in osteogenic differentiation has been proved [14]. For further investigation of the mechanism by which miR-138-5p inhibited osteogenic differentiation of hAVICs, Wnt/ $\beta$ catenin signaling pathway-related proteins were detected. According to the results, overexpression of miR-138-5p contributed to reduce Wnt5a and $\beta$-catenin protein expression, while miR-138-5p knockdown achieved the opposite outcomes (Fig. 4A). Simultaneous overexpression of RUNX2 and miR-138-5p could reversed the inhibitory effect of overexpressed miR-138-5p on Wnt5a and $\beta$-catenin (Fig. 4B). The above results illustrated that the inhibitory effect of miR-138-5p on osteogenic differentiation was dependent on the $\mathrm{Wnt} / \beta$-catenin signaling pathway.

\section{Discussion}

CAVD is one of the main causes of death and morbidity of cardiovascular diseases, especially in the elderly, and development of this disease is associated with osteogenic differentiation (or calcification). However, there are no specific drugs effective for CAVD from lack of insight into the pathomechanism of osteogenic differentiation. Aortic valve replacement is currently the only means to alleviate the condition. But in recent years, it has been reported that osteogenic differentiation also occurs in the surgically implanted aortic valves, and AVIC, the main cell of aortic valve tissue, is also involved in this pathological process. Therefore, for treating CAVD, there is a need for an investigation on the mechanism of aortic valve calcification [15]. In this study, we found that miR138-5p negatively regulates the process of osteogenic differentiation of human calcified aortic valve by targeting RUNX2.

Among miRNAs, miR-138-5p has gradually received attention in the regulation of osteogenic differentiation. For example, hsa-miR-138 inhibits adipogenic 


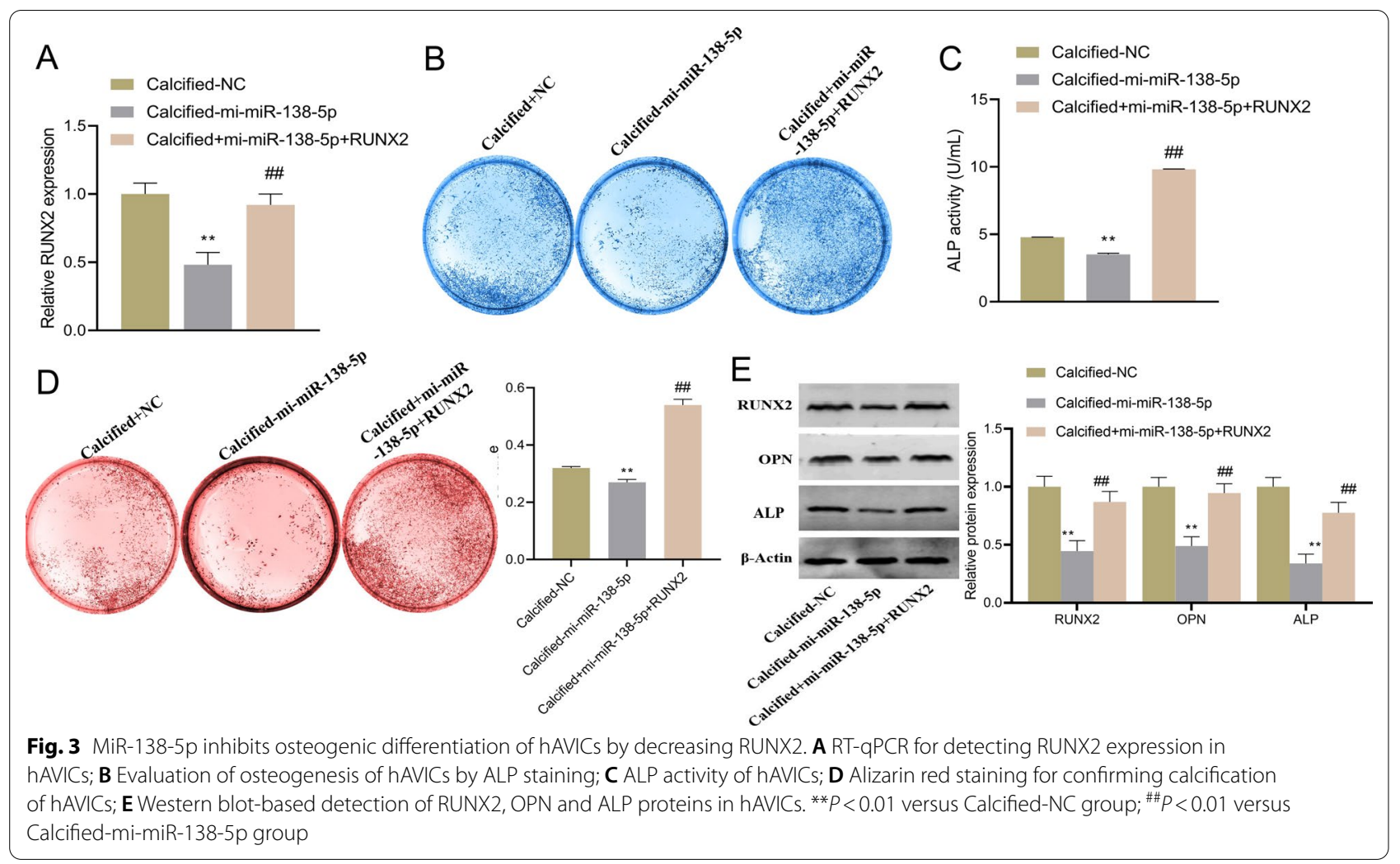

differentiation of adipose tissue-derived hMSCs through adenovirus EID-1 [16], and down-regulation of Noggin and miR-138 synergistically promote osteogenic transformation of MSCs [17]. In our study, miR-138-5p was down-regulated in CAVD patients, and was downregulated during osteogenic differentiation of hAVICs. OPN, a highly phosphorylated glycoprotein secreted by osteoblasts, can be deposited into the bone matrix to promote osteocyte adhesion; OPN is one of the phenotypes of osteoblasts, and participates in resorption, mineralization of bone matrix and in maintenance of bone tissue integrity [18]. ALP, a marker of osteoblast, can be adopted for detecting the degree of differentiation of osteoblasts [19]. In this study, we also found that up-regulation of miR-138-5p inhibited OPN and ALP protein expression and ALP activity, and at the same time, and ALP staining and alizarin red staining also indicated that overexpressed miR-138-5p inhibited osteogenic differentiation of hAVICs.

RUNX2 is a core molecule in osteogenic differentiation [20-23], which can be down-regulated by miR-204-5p to inhibit osteogenic differentiation in rat BMSC. According to the results in this study, both protein and transcript levels of RUNX2 were significantly increased in hAVICs from patients with calcified aortic valve and presented a negative correlation with miR138-5p. RUNX2 was also confirmed to be a target gene of miR-138-5p, and the inhibitory effect of miR-138-5p on osteogenic differentiation of hAVICs was achieved through RUNX2.

Wnt/ $\beta$-catenin pathway is reported to be one of the essential pathways for the osteogenic process [14]. Activation of Wnt promotes AVICs to express $\beta$-catenin and resulting promotion of transcription and expression of RUNX2 and ultimately osteogenic differentiation of progenitor cells and calcification of vascular smooth muscle cells $[24,25]$. However, the relationship between miR-138-5p and Wnt/ $\beta$-catenin pathway in CAVD is not clear. It has been demonstrated that protein levels of canonical Wnt/B-catenin signaling pathway are markedly changed in human patients, rodent models, and in vitro valve interstitial cells $[15,26,27]$. In the present study, overexpression of miR-138-5p significantly inhibited the expression of Wnt/ $\beta$-catenin signaling pathway-associated proteins, while simultaneous overexpression of miR-138-5p and RUNX2 reversed this inhibitory effect, suggesting that the $\mathrm{Wnt} / \beta$-catenin signaling pathway was involved in the regulation of miR-138-5p/RUNX2 on osteogenic differentiation. 


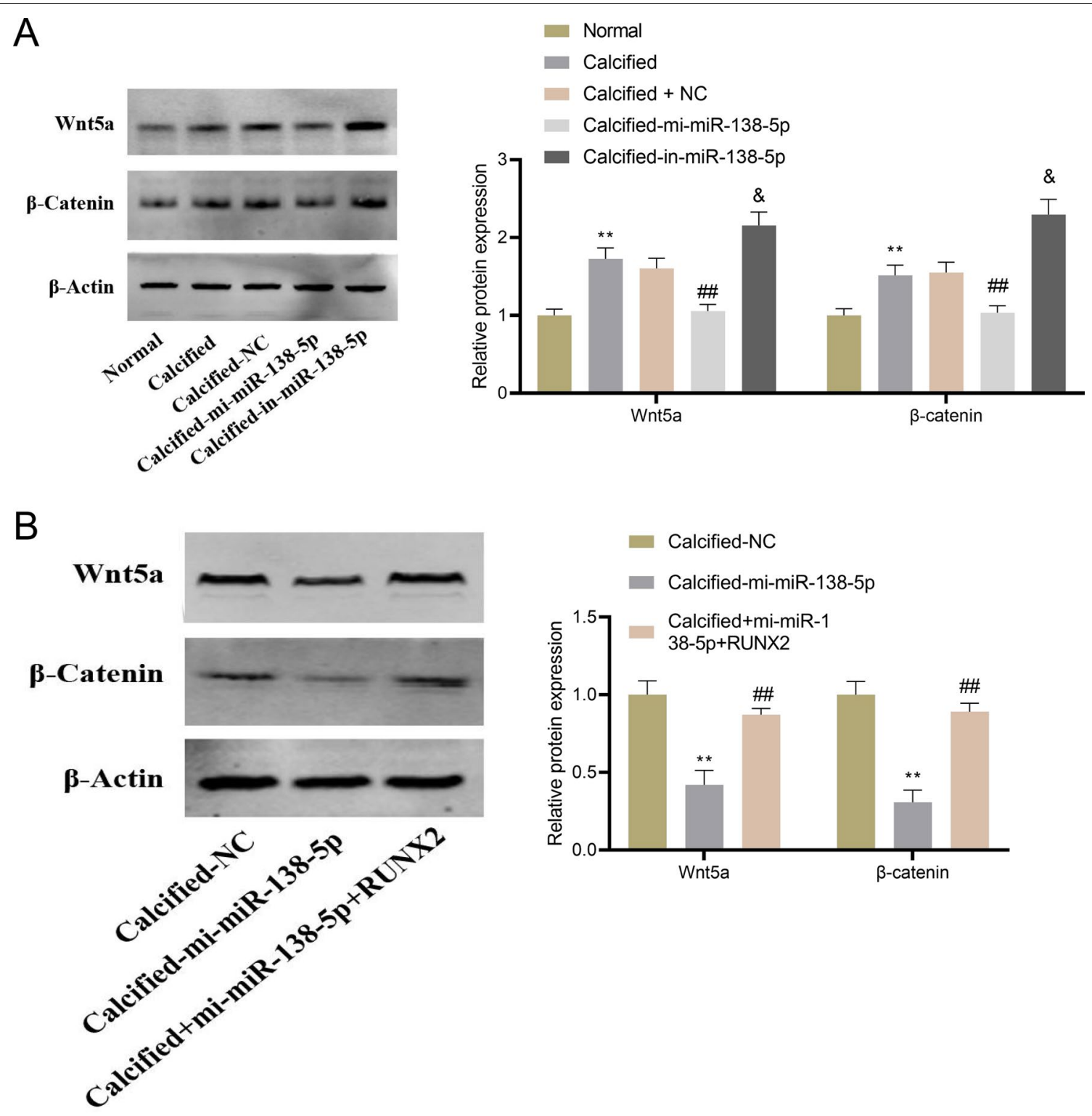

Fig. 4 Effect of miR-138-5p targeting RUNX2 on Wnt/ $\beta$-catenin signaling pathway. A Western blot-based detection of Wnt 5 a and $\beta$-catenin protein expression in osteogenically differentiated hAVICs transfected with miR-138-5p mimics or inhibitor. ${ }^{* *} P<0.01$ versus Normal group; ${ }^{\# \#} P<0.01$ versus Calcified group; $\& P<0.05$ versus Calcified-NC group. B Western blot-based detection of Wnt 5 a and $\beta$-catenin protein expression in osteogenically differentiated hAVICs transfected with miR-138-5 mimics or miR-138-5p mimics combined with RUNX2 overexpression plasmid

\section{Conclusion}

In summary, through RUNX2 and Wnt/ $\beta$-catenin signaling pathway, miR-138-5p acts as a positive regulator of osteogenic differentiation in CAVD patients to involve in inhibiting valve calcification. This miRNA may become a new marker of osteogenic differentiation, contributing to a potential therapeutic strategy for preventing or treating to diseases associated with valve calcification.

\section{Supplementary Information}

The online version contains supplementary material available at https://doi. org/10.1186/s12872-022-02471-6.

Additional file 1. All uncut Western blot bands in the article.

Acknowledgements

Not applicable. 


\section{Authors' contributions}

YF contributed to conception and design of the study. QH, WZ performed the statistical analysis. YF wrote the first draft of the manuscript. QH, WZ, TW, DD and LS wrote sections of the manuscript. All authors contributed to manuscript revision, read, and approved the submitted version.

\section{Funding}

This research did not receive any funding

\section{Availability of data and materials}

The data used to support the findings of this study are available from the corresponding author upon request.

\section{Declarations}

\section{Ethics and consent to participate}

This study was approved by the Medical Ethics Committee of The First Affiliated Hospital of Xinjiang Medical University (K202107-09). And informed consent was obtained from all subjects. The study was conducted in accordance with the Declaration of Helsinki (as revised in 2013).

\section{Consent for publication}

Not applicable.

\section{Competing interests}

The authors claim that there is no conflict of interest between them.

\section{Author details}

${ }^{1}$ Department of Cardiac Surgery, The First Affiliated Hospital of Xinjiang Medical University, 137 Liyushan South Road, Xinshi District, Urumqi 830054, Xinjiang, China. ${ }^{2}$ Department of Cardiology, The First Affiliated Hospital of Xinjiang Medical University, 137 Liyushan South Road, Xinshi District, Urumqi 830054, Xinjiang, China.

Received: 14 September 2021 Accepted: 21 January 2022

Published online: 02 February 2022

\section{References}

1. Ossareh S. Cardiac valvular calcification in hemodialysis patients. Iran J Kidney Dis. 2013;7:77-9.

2. Zhang M, Liu X, Zhang X, Song Z, Han L, He Y, et al. MicroRNA-30b is a multifunctional regulator of aortic valve interstitial cells. J Thorac Cardio vasc Surg. 2014;147:1073-1080.e1072.

3. Towler DA. Molecular and cellular aspects of calcific aortic valve disease Circ Res. 2013;113:198-208.

4. Sánchez-Perales C, Vázquez Ruizde Castroviejo E, García-Cortés MJ, Biechy Mdel M, Gil-Cunquero JM, Borrego-Hinojosa J, et al. Valvular calcifications at the start of dialysis predict the onset of cardiovascular events in the course of follow-up. Nefrologia. 2015;35:157-63.

5. Seok HY, Chen J, Kataoka M, Huang ZP, Ding J, Yan J, et al. Loss of MicroRNA-155 protects the heart from pathological cardiac hypertrophy. Circ Res. 2014;114:1585-95.

6. Yang X, Qin Y, Shao S, Yu Y, Zhang C, Dong H, et al. MicroRNA-214 inhibits left ventricular remodeling in an acute myocardial infarction rat model by suppressing cellular apoptosis via the phosphatase and tensin homolog (PTEN). Int Heart J. 2016;57:247-50.

7. Lu X, Yin D, Zhou B, Li T. MiR-135a promotes inflammatory responses of vascular smooth muscle cells from $\mathrm{db} / \mathrm{db}$ mice via downregulation of FOX01. Int Heart J. 2018;59:170-9.

8. Hayes J, Peruzzi PP, Lawler S. MicroRNAs in cancer: biomarkers, functions and therapy. Trends Mol Med. 2014;20:460-9.

9. Fang M, Wang CG, Zheng C, Luo J, Hou S, Liu K, et al. Mir-29b promotes human aortic valve interstitial cell calcification via inhibiting TGF- $\beta 3$ through activation of wnt3/ $\beta$-catenin/Smad3 signaling. J Cell Biochem. 2018;119:5175-85.

10. Jiao W, Zhang D, Wang D, Xu R, Tang L, Zhao M, et al. MicroRNA-638 inhibits human aortic valve interstitial cell calcification by targeting Sp7. J Cell Mol Med. 2019;23:5292-302.
11. Eskildsen T, Taipaleenmäki H, Stenvang J, Abdallah BM, Ditzel N, Nossent AY, et al. MicroRNA-138 regulates osteogenic differentiation of human stromal (mesenchymal) stem cells in vivo. Proc Natl Acad Sci USA. 2011;108:6139-44.

12. Zhang L, Liu Y, Feng B, Liu LG, Zhou YC, Tang H. MiR-138-5p knockdown promotes osteogenic differentiation through FOXC1 upregulation in human bone mesenchymal stem cells. Biochem Cell Biol. 2021;99:296-303

13. Chen JH, Simmons CA. Cell-matrix interactions in the pathobiology of calcific aortic valve disease: critical roles for matricellular, matricrine, and matrix mechanics cues. Circ Res. 2011;108:1510-24.

14. Wang Y, Zhang X, Shao J, Liu H, Liu X, Luo E. Adiponectin regulates BMSC osteogenic differentiation and osteogenesis through the Wnt/ $\beta$-catenin pathway. Sci Rep. 2017;7:3652.

15. Zeng Q, Song R, Ao L, Weyant MJ, Lee J, Xu D, et al. Notch1 promotes the pro-osteogenic response of human aortic valve interstitial cells via modulation of ERK1/2 and nuclear factor-kB activation. Arterioscler Thromb Vasc Biol. 2013;33:1580-90.

16. Yang Z, Bian C, Zhou H, Huang S, Wang S, Liao L, et al. MicroRNA hsamiR-138 inhibits adipogenic differentiation of human adipose tissuederived mesenchymal stem cells through adenovirus EID-1. Stem Cells Dev. 2011;20:259-67.

17. Sun XK, Zhou J, Zhang L, Ma T, Wang YH, Yang YM, et al. Down-regulation of Noggin and miR-138 coordinately promote osteogenesis of mesenchymal stem cells. J Mol Histol. 2017:48:427-36.

18. Foster BL, Ao M, Salmon CR, Chavez MB, Kolli TN, Tran AB, et al. Osteopontin regulates dentin and alveolar bone development and mineralization. Bone. 2018;107:196-207.

19. Ma J, Wang Z, Zhao J, Miao W, Ye T, Chen A. Resveratrol attenuates lipopolysaccharides (LPS)-induced inhibition of osteoblast differentiation in MC3T3-E1 cells. Med Sci Monit. 2018:24:2045-52.

20. Grim JC, Aguado BA, Vogt BJ, Batan D, Andrichik CL, Schroeder ME, et al. Secreted factors from proinflammatory macrophages promote an osteoblast-like phenotype in valvular interstitial cells. Arterioscler Thromb Vasc Biol. 2020;40:e296-308

21. Yang N, Wang L, Chen T, Liu R, Liu Z, Zhang L. ZNF521 which is downregulated by miR-802 suppresses malignant progression of hepatocellular carcinoma through regulating Runx2 expression. J Cancer. 2020;11:5831-9.

22. Lim KE, Park NR, Che $X$, Han MS, Jeong JH, Kim SY, et al. Core binding factor $\beta$ of osteoblasts maintains cortical bone mass via stabilization of Runx2 in mice. J Bone Miner Res. 2016:31:245.

23. Le MH, Warotayanont R, Stahl J, Den Besten PK, Nakano Y. Amelogenin Exon4 forms a novel miRNA that directs ameloblast and osteoblast differentiation. J Dent Res. 2016;95:423-9.

24. Albanese I, Yu B, Al-Kindi H, Barratt B, Ott L, Al-Refai M, et al. Role of noncanonical wnt signaling pathway in human aortic valve calcification. Arterioscler Thromb Vasc Biol. 2017;37:543-52.

25. Caira FC, Stock SR, Gleason TG, McGee EC, Huang J, Bonow RO, et al. Human degenerative valve disease is associated with up-regulation of low-density lipoprotein receptor-related protein 5 receptor-mediated bone formation. J Am Coll Cardiol. 2006;47:1707-12.

26. Zhou W, Huang H, Zhu H, Zhou P, Shi X. New metabolites from the biotransformation of ginsenoside Rb1 by Paecilomyces bainier sp.229 and activities in inducing osteogenic differentiation by Wnt/ $\beta$-catenin signaling activation. J Ginseng Res. 2018;42:199-207.

27. Wang Y, Zhou CJ, Liu Y. Wnt signaling in kidney development and disease. Prog Mol Biol Transl Sci. 2018;153:181-207.

\section{Publisher's Note}

Springer Nature remains neutral with regard to jurisdictional claims in published maps and institutional affiliations. 